Management Forschung und Praxis

Universität Konstanz

herausgegeben von Prof. Dr. Rüdiger G. Klimecki

Markus Gmür

\title{
Strategisches Management für \\ Nonprofit-Organisationen
}

Nr. 28 (1999)

Eine textähnliche Version erscheint in:

Stefan Nährlich/Annette Zimmer (Hrsg.) Management in Nonprofit-Organisationen: Eine praxisorientierte Einfuihrung. Opladen: Leske \& Budrich 1999

Markus Gmür, Dr. rer. soc.

Lehrstuhl für Management

Fakultät für Verwaltungswissenschaft

Universität Konstanz

Postfach D 93

D-78457 Konstanz

Tel. (07531) 882493

e-mail:_markus.gmuer@uni-konstanz.de 


\begin{abstract}
:
Strategien sind die langfristigen Handlungsprogramme einer Organisation im Wettbewerb um knappe Ressourcen. Das gilt unabhängig davon, ob der Zweck der Organisation in der Gewinnmaximierung oder der Verwirklichung gesellschaftspolitischer Ziele besteht. Dementsprechend erstreckt sich der Ressourcenwettbewerb von der Finanzierung über das Engagement und die Fachexpertise der Organisationsmitglieder bis zur Anerkennung bzw. Unterstützung durch Kunden, Leistungsempfänger oder politische Entscheidungsträger.

Strategisches Management umfasst den gesamten Prozess der Strategieentwicklung von der Klärung der grundlegenden Ziele und der Analyse der aktuellen Ausgangslage bis zur Erarbeitung eines

Maßnahmenplans zu ihrer Realisierung. Dazu liegen eine ganze Reihe von Konzepten und Instrumenten vor, die im Zusammenhang marktwirtschaftlicher Unternehmensführung entwickelt wurden, aber auch für die langfristig angelegte Führung und Leitung von Nonprofit-Organisationen nutzbar gemacht werden können.

Strategien dienen dazu, den langfristigen Bestand und die fortlaufende Entwicklungsfähigkeit einer Organisation zu gewährleisten, damit sie ihre Ziele auch unter wechselnden Bedingungen erreichen kann.
\end{abstract}

\title{
Inhalt
}

1 Merkmale und Funktionen des Strategischen Management ................................................ 3

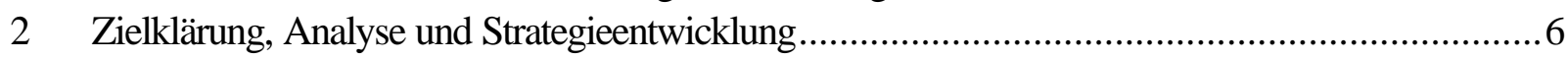

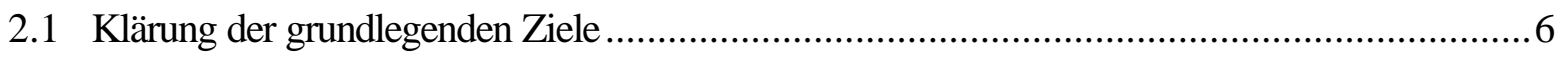

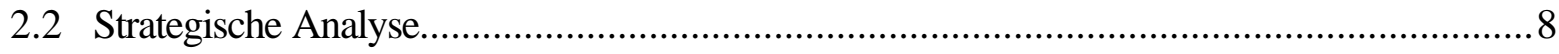

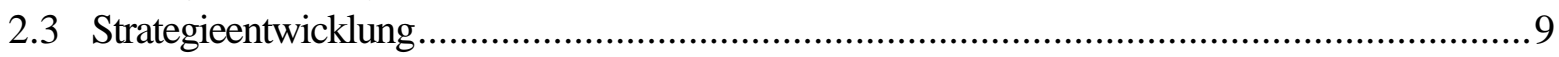

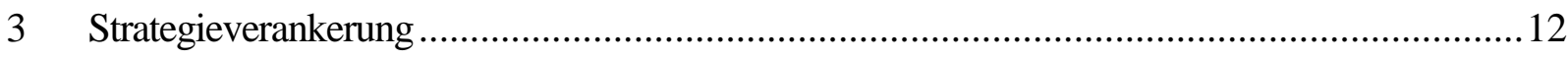

3.1 Die Rolle der Führungskräfte im Strategischen Management ...........................................13

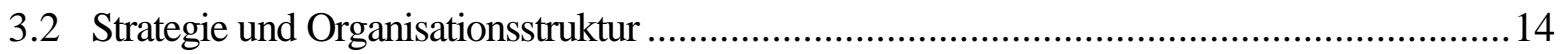

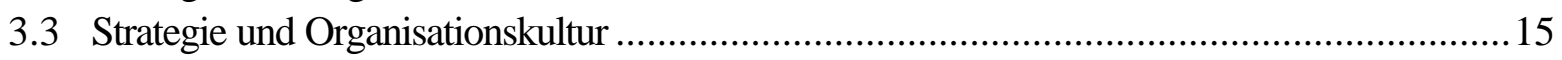

$4 \quad$ Strategisches Controlling und Entwicklungsfähigkeit ................................................... 17

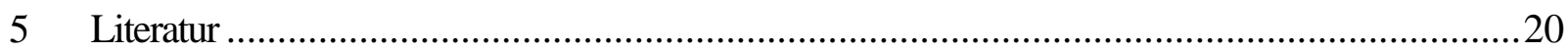




\section{Merkmale und Funktionen des Strategischen Management}

Strategisches Management ist "in". Nachdem die Zahl der Konzepte und Publikationen zur strategischen Unternehmensführung in den vergangenen 30 Jahren stetig gewachsen ist, erschließt sich nun auch der Nonprofit-Sektor diese Kernfunktion des Managements. Wie die Untersuchung von Middleton-Stone und Crittenden (1993) gezeigt hat, steht derzeit noch die Übertragung bewährter Modelle aus dem ForProfit-Bereich im Vordergrund. Die Besonderheiten von Nonprofit-Organisationen mit ihren Konsequenzen für das Strategische Management sind dagegen noch wenig untersucht worden. $\mathrm{Zu}$ diesen Besonderheiten zählen die Autoren die Einflüsse von Interessengruppen (Stakeholder) und dem Board sowie die Schwierigkeiten bei der Erfolgsmessung und der Strategieumsetzung unter der Voraussetzung basisdemokratischer Strukturen. Einige dieser Besonderheiten von NonprofitOrganisationen werden in diesem Beitrag erörtert.

Strategisches Management beruht auf einer bestimmten Art und Weise, wie Leitungsprobleme wahrgenommen und Entscheidungen getroffen werden. Strategisch denken und handeln heißt:

- langfristig wirksame Effekte anzustreben und demgegenüber die Lösung von Problemen, die nur von vorübergehender Bedeutung sind, zurückzustellen.

- antizipativ zu denken, d.h. Entwicklungen, die sich gegenwärtig abzeichnen, gedanklich weiterzuverfolgen und die Entscheidungen an zukünftig abzusehenden Ereignissen auszurichten.

- zu integrieren, d.h. in Hinblick auf ein strategisches Ziel alle relevanten Funktionen und Ressourcen aufeinander abzustimmen und keine isolierten Detaillösungen zu verfolgen.

- Ressourcen selektiv und konzentriert auf die wesentlichen strategischen Ziele auszurichten und nicht $\mathrm{zu}$ versuchen, alle möglichen Optionen gleichzeitig zu verfolgen und damit die Ressourcen aufzusplittern. Strategisches Denken bedeutet, immer wieder einmal "nein" zu sagen, damit man dort, wo es wirklich wichtig ist, mit gutem Gewissen "ja" sagen kann.

- nach Wettbewerbsvorteilen zu streben. Diese wichtige Eingrenzung wird häufig übersehen. Eine langfristige Planung oder eine Konzentration auf Schwerpunkte kann dazu dienen, politische Ziele zu verwirklichen oder einfach Budgetrestriktionen zu genügen. Strategisch wird sie, wenn die Organisation sich dabei gegen Wettbewerber in der Zielverfolgung oder Konkurrenten um die Mittelvergabe langfristig durchsetzen muss. Dementsprechend geben Strategiekonzepte auch keine absoluten sondern relative Empfehlungen. Wie kann ich erfolgreicher als meine Konkurrenten werden?

Als zentraler Orientierungspunkt hat sich in der Strategielehre das Konstrukt des Strategischen Erfolgspotentials durchgesetzt. Der Begriff steht für die Chance einer Organisation, sich gegenüber den Konkurrenten einen bedeutenden Vorteil zu verschaffen, weil sie zwei Merkmale aufweist:

- Erstens stellt es einen langfristig relevanten Nutzen für eine wesentliche Stakeholdergruppe (z.B. Kunden oder Geldgeber) dar, um deren Unterstützung die Organisation sich bemüht.

- Zweitens ist es nicht substituierbar, oder seine Nachahmung erfordert zumindest einen hohen finanziellen und zeitlichen Aufwand. 
Strategische Erfolgspotentiale können in den Leistungen der Organisation begründet sein, die sie allein oder auf besondere Weise erbringt. Unabhängig vom Leistungsangebot können sie aber auch in den Leistungserbringern, d.h. einem besonders motivierten Mitarbeiterstab, in der Organisationskultur oder in bestehenden Beziehungen zu wichtigen Stakeholders bestehen. Im Umweltschutzbereich hat sich beispielsweise Greenpeace ein Aktivistenimage aufgebaut, das offensichtlich den Wertvorstellungen jüngerer Fördermitglieder sehr gut entspricht. Unter den Hilfsorganisationen verfügt das Rote Kreuz über weltweit akzeptierte Symbole (Kreuz bzw. Halbmond) mit einer langen Tradition. Strategische Erfolgspotentiale können vielfältig sein, und ihre Bedeutung ist eng an das Tätigkeitsfeld der jeweiligen Organisation gebunden.

Neben der Hauptfunktion der langfristigen Bestandssicherung hat das Strategische Management für eine Organisation vielfältige Nebenfunktionen:

- Koordinationsfunktion: Strategisches Management baut auf Zielen auf, die als Richtschnur vielfältigster Entscheidungen wirken. Aus ihnen lassen sich Prioritäten für den Ressourceneinsatz ableiten.

- Evaluationsfunktion: Die Strategischen Ziele können als Grundlage einer langfristigen Erfolgsbeurteilung einer Organisation und der Tätigkeit ihrer Träger dienen.

- Reflexions- und Klärungsfunktion: Strategisches Management hält dazu an, mögliche Ziele und die daraus folgenden Konsequenzen auszuloten.

- Integrationsfunktion: Strategisches Management bietet Anlässe dafür, sich über Ziele und Grundsätze zu verständigen, eine Verständigungs- und Vertrauensbasis zu schaffen und damit die gegenseitige Abstimmung in der Alltagsarbeit zu erleichtern.

Insbesondere die große Bedeutung der beiden letzten Funktionen wird häufig unterschätzt, wenn etwa die Strategische Planung unter Hinzuziehung externer Berater auf die Organisationsspitze konzentriert wird. Damit werden wichtige Impulse von der Organisationsbasis ausgeschlossen, wo sich eine misstrauische Stimmung gegenüber der Spitze entwickeln kann.

Auch wenn man davon ausgehen kann, dass sich Erkenntnisse und Konzepte des Strategischen Management ohne weiteres auf Nonprofit-Organisationen in Wettbewerbssituationen anwenden lassen, sind einige Besonderheiten dieses Organisationstyps für ihre sachgerechte Anwendung in der Praxis zu berücksichtigen. Nutt und Backoff (1992: 22ff.) weisen in diesem Zusammenhang auf folgende drei Aspekte hin:

- Legitimierung und Finanzierung: Nonprofit-Organisationen sind häufig von einer Vielzahl von Finanzierungsquellen mit unterschiedlichen Interessen abhängig, die sie langfristig zum Ausgleich bringen müssen. Damit rückt das Stakeholder-Management in den Mittelpunkt von Organisationsstrategien. Nutt und Backoff bezeichnen die Aufrechterhaltung finanzieller Arrangements als den wesentlicher Kern der Strategischen Aufgabe.

- Zielvielfalt und -mehrdeutigkeit: Die Ziele von Nonprofit-Organisationen lassen sich in aller Regel nicht auf einfache Kriterien ökonomischen Erfolgs reduzieren. Ertrags- und Effizienzziele sind immer nur Mittel zur Realisierung eines übergeordneter Zwecks, der aber seinerseits nur schwer bestimmbar ist. Für einen Umweltschutzverband ist es ebenso wenig sinnvoll, sich zum Ziel zu setzen (und sich 
anschließend daran messen zu lassen!), den Grad der Umweltverschmutzung zu reduzieren, wie für einen Wohlfahrtsverband, die Anzahl armer Familien in Deutschland zu senken. NonprofitOrganisationen müssen sich deshalb mit mehrdeutigen und nur schwer messbaren Zielen begnügen. Diese "weichen" Ziele lassen sich fixieren, indem sie im Konsens ihrer Mitglieder verankert werden. Somit wird über ihr Erreichen eine gemeinsame Vorstellung erzeugt: "Wenn wir mit medienwirksamen Aktionen ein paar Umweltsünder treffen, schrecken wir andere ab und tragen dazu bei, das Umweltbewusstsein in der Öffentlichkeit zu stärken." "Wenn es uns gelingt, Obdachlose von der Straße zu bringen, leisten wir damit einen Beitrag zur Lösung eines großen sozialen Problems."

- Tradition als Wert: Wie Salipante und Golden-Biddle (1995) gezeigt haben, haben Tradition und eine kontinuierliche Entwicklung für Nonprofit-Organisationen eine größere Bedeutung als im For-ProfitBereich. Sie ist eine wesentliche Grundlage für Vertrauen in und Identifikation mit der Organisation. Entgegen den Grundsätzen einer marktorientierten Strategielehre hat die Sicherung von Kontinuität auch bei erheblichen Veränderungen der äußeren Situation eine hohe Priorität. Die beiden Autoren erklären das damit, dass die Stakeholders vieler Nonprofit-Organisationen über soziale Bedürfnisse oder Weltbilder an sie gebunden sind. Diese Bedürfnisse lassen weniger Flexibilität zu als das Nutzenkalkül, auf dem Unternehmen vorrangig beruhen.

Die ersten beiden Merkmale erhöhen die Komplexität des Strategischen Management, wie es unter marktwirtschaftlichen Bedingungen praktiziert wird, weil sie differenziertere Konzepte erfordern. Das letzte Merkmal beschränkt den Anwendungsbereich solcher Konzepte. Je größer in einer Organisation die Bedeutung ihrer tradierten Grundsätze ist, um so geringer ist der Spielraum strategischer Überlegungen und der daraus abgeleiteten Handlungsprogramme. Eine Organisation strategisch zu führen, macht es erforderlich, alte Traditionen aufs Spiel zu setzen. Der Strategieentwicklungsprozess eröffnet aber die Chance, eine neue Tradition zu begründen

Der Prozess des Strategischen Management lässt sich in fünf Abschnitte unterteilen, von der Klärung der grundlegenden Ziele bis zu den konkreten Maßnahmen und ihrer abschließenden Evaluation.

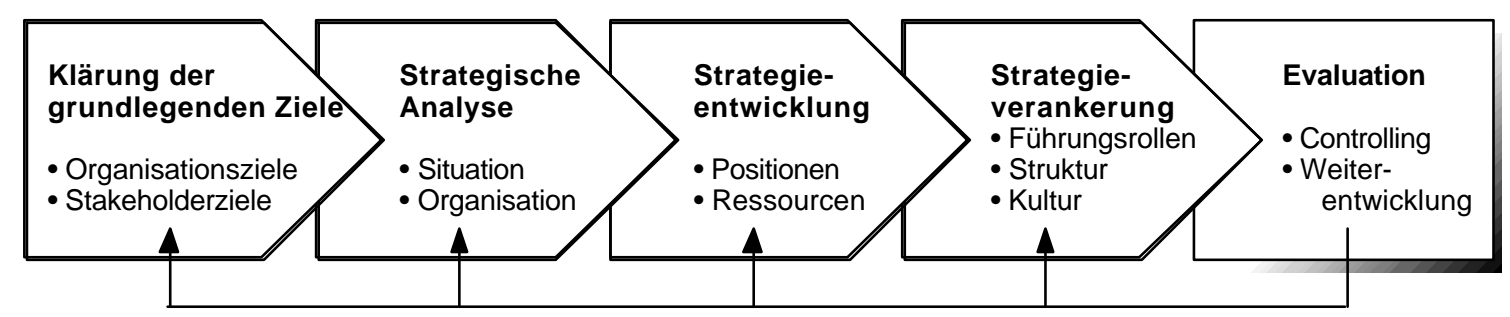

Die Evaluationsphase ist gleichzeitig Abschluss eines Strategieprozesses und Grundlage für die nachfolgenden Prozesse, die durch eine Zielklärung, eine Analysephase, eine Überprüfung der Strategien oder neuerliche Maßnahmen der Strategieverankerung eingeleitet werden können. Die einzelnen Phasen werden in den folgenden drei Kapiteln näher erörtert. 


\section{Zielklärung, Analyse und Strategieentwicklung}

\subsection{Klärung der grundlegenden Ziele}

Strategien dienen dazu, die wesentlichen langfristigen Ziele, auf denen eine Organisation beruht, zu erreichen. Eine Klärung dieser grundlegenden Ziele geht dem Strategischen Management voraus. Obwohl sich das Ziel als konstituierendes Merkmal jeder Organisation prinzipiell beliebig bestimmen lässt, folgt es in der Praxis typischen Mustern. In marktwirtschaftlichen Unternehmen geht man traditionell davon aus, das wesentliche Ziel einer Organisation sei die Verwirklichung einer unternehmerischen Idee: Gottlieb Daimler verfolgte die Idee, eine Kutsche ohne Pferde zu entwickeln, woraus ein Automobil wurde. Jobs und Wozniak hatten sich in den 70er Jahren zum Ziel gesetzt, gegen die zentralen Großrechner einen unabhängigen Kleinrechner für jedermann zu setzen; ihr Apple Macintosh läutete die Ära der Personalcomputer ein. Mit der zunehmenden öffentlichen Wahrnehmung der privaten Unternehmen setzte sich langsam die Erkenntnis durch, dass Strategien nicht nur unternehmerische Ideen verkörpern, sondern auch den Zielen der wesentlichen Stakeholders entsprechen sollen. Diese Legitimationsbasis verengt sich im Ansatz des Shareholder Value der 90er Jahre wiederum auf eine einzige Gruppe, nämlich die der Kapitalgeber: Strategisches Management wird so ausgerichtet, dass es die Eigenkapitalrendite bzw. den Börsenwert maximiert. Unternehmensstrategien bewegen sich also in einem Spannungsfeld zwischen unternehmerischer Vision, gesellschaftlichem Interessenausgleich und Gewinnmaximierung.

Auch das Strategische Management von Nonprofit-Organisationen bewegt sich in einem Spannungsfeld zwischen zwei Polen: Den einen Pol bilden die Ziele der Stakeholders, den anderen die Ziele der Organisation selbst.

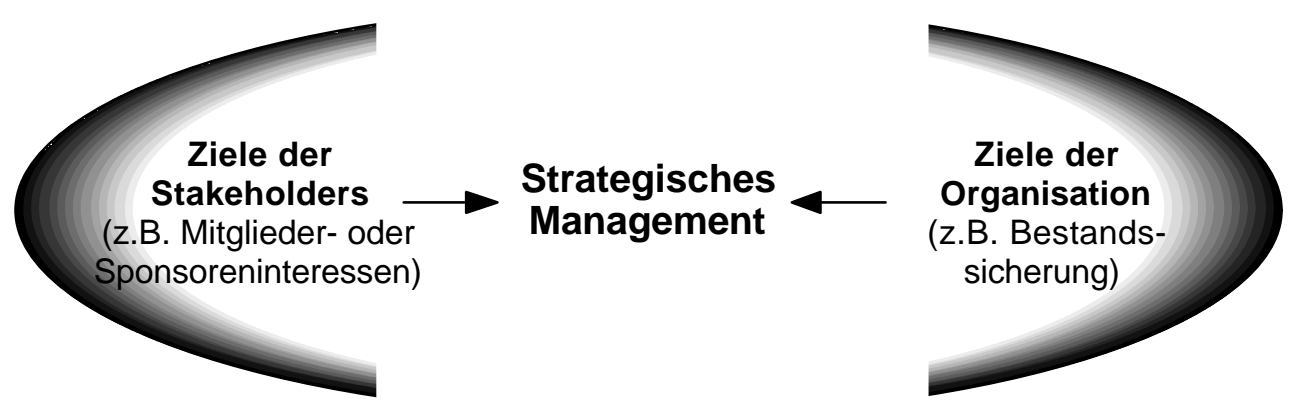

Ziele der Stakeholders sind ein möglicher Ausgangspunkt des Strategischen Management. Mit dem Begriff Stakeholder werden alle Personen oder Gruppen bezeichnet, die ein spezifisches Interesse an der Organisation und ihren Zielen haben und deshalb auf die Aktivitäten der Organisation reagieren. Stakeholders sind beispielsweise die Basis (aktive und passive Mitglieder), der Vorstand und andere Gremien, angestelltes Personal und ihre Vertretung, Zulieferer und Leistungsempfänger der Organisation, aber auch übergeordnete Verbände, politische Institutionen, Konkurrenten und Kooperationspartner oder die Medien. Hauptaufgabe des Stakeholder-Management in einer Nonprofit-Organisation ist die Ermittlung und Bewertung der Einflüsse von Seiten der Stakeholders. Diese sind von mehreren Faktoren abhängig (Horak 1996): 
- Grundsätzliche Einstellung gegenüber der Organisation: z.B. unterstützend oder ablehnend, aktiv oder passiv.

- Einflussstärke auf die Organisation: z.B. durch finanzielle Abhängigkeit oder Kontakte zu wichtigen Entscheidungsstellen.

- Fähigkeit zur Artikulation der Einstellungen: z.B. über Medien oder eigene Informationsveranstaltungen.

- Organisationsgrad der Stakeholders: z.B. in eigenen Interessenverbänden oder durch gegenseitige Absprachen.

- Einflussmöglichkeiten der Organisation: z.B. durch Aufklärungsarbeit.

- Nutzenzuwachs durch die Organisation: z.B. durch unmittelbare Leistungen oder indirekte Effekte aus erfolgreichen Aktivitäten in der Öffentlichkeit.

Daraus ergibt sich gegenüber jeder Gruppe eine Abhängigkeitsbilanz (Horak 1996: 99). Sie lässt Schlussfolgerungen darüber zu, inwieweit die Organisation autonom agieren und ihre grundlegenden Ziele frei bestimmen kann.

Stakeholder-Ziele müssen zwar nicht, können aber im Widerspruch zu den Zielen der Organisation stehen. Wird ein Verein oder Verband neu gegründet, fließen die Ziele der Gründungsmitglieder unmittelbar ein. Mit der Zeit entwickeln Organisationen eine eigene Identität, die von einem Kreis aktiver Mitglieder oder dem Vorstand ist und sich von der breiten Basis zunehmend ablöst. Das ist ein Effekt der Institutionalisierung jeder Organisation. Dadurch kann es zu einer Entfremdung kommen wie beispielsweise im Fall von Greenpeace Deutschland, als der Vorstand das Wirtschaftsberatungsunternehmen Roland Berger der Deutschen Bank mit einem Reorganisationsprojekt beauftragte, was nach kurzer Zeit zum Austritt von Basisgruppen führte. Die Ziele der Organisation orientieren sich am Interesse der Organisation selbst und können im Extremfall den Zielen wichtiger Stakeholders widersprechen. Die Erhaltung einer Organisation (sogar als Selbstzweck, wenn auch kaschiert) kann zur eigenen Legitimationsbasis von Organisationsstrategien werden. Wirtschafts- ebenso wie Umweltverbände und Parteien ebenso wie Kirchen haben Phasen durchlaufen, in denen für den ursprünglichen Mitgliederkreis deutlich wurde, wie sich die Organisation mit ihrem Vorstand und dem angestellten Personal von der Basis ablöste. Aus der Perspektive des Organisationskerns kann es sinnvoll erscheinen, Wachstum anzustreben, das Auftreten nach außen zu verändern, sich aus bisherigen Aktionsfeldern zurückzuziehen oder ganz neue Ziele zu verfolgen, während die Basis vielleicht kein Wachstum wünscht oder bestimmte Ziele aus grundsätzlichen Überlegungen ablehnt.

$\mathrm{Ob}$ eine Organisation eigene Ziele (entsprechend einer unternehmerischen Vision) entwickelt oder sie aus einem Konsens, einem Kompromiss oder einer Synthese von Stakeholder-Interessen ableitet, hängt wesentlich davon ab, wie sie ihre Abhängigkeitsbilanz interpretiert. Je größer die eingeschätzte Abhängigkeit ist, um so eher wird nach einem Ausgleich mit bestimmten Stakeholder-Interessen gestrebt. Diese Einschätzung ist aber wiederum stark abhängig vom Selbstbewusstsein der entscheidenden Organe und Personen. 
Ergebnis dieser ersten Phase im Prozess des Strategischen Management ist ein Katalog der grundlegenden Ziele. Sie sollten möglichst klar, eindeutig und widerspruchsfrei sein, damit sie strategisch realisierbar sind.

\subsection{Strategische Analyse}

Zwischen der Zielbildung und ihrer Umsetzung in Strategien steht die Phase der Strategischen Analyse. Sie erstreckt sich auf zwei große Bereiche und dient dazu, Möglichkeiten und Grenzen potentieller strategischer Alternativen zu bestimmen.

Die Situationsanalyse umfasst alle Felder außerhalb der Organisation, aus denen sich Chancen und Gefahren für ihre zukünftigen Aktivitäten ergeben können. Mit ihr werden die aktuelle Position der Organisation im Wettbewerb, ihre Rahmenbedingungen und mögliche zukünftige Veränderungen erfasst. Neben einer eingehenden Stakeholder-Analyse (Horak 1996) können Informationen aus allen Bereichen der Sozialforschung herangezogen werden oder gar eigene gezielte Befragungen durchgeführt werden. Menschenrechtsorganisationen werden sich intensiv mit weltpolitischen Entwicklungen befassen, Verbraucherschutzorganisationen mit neuen Verfahren der Lebensmittelverarbeitung und politische Parteien mit demographischen Daten. Je höher die eingeschätzte Risikolage ist, um so größer ist die Bedeutung solcher Informationen und entsprechend größer die Neigung, dafür Zeit und Geld aufzuwenden. Empirische Untersuchungen deuten an, dass eine intensivere Situationsanalyse nicht nur zu einer selbstsicheren Situationseinschätzung und größerem Mut zu strategischen Entscheidungen führt, sondern sich letztlich auch positiv auf den Erfolg einer Organisation auswirkt (Thomas et al. 1993).

Die Organisationsanalyse bezieht sich auf die Ressourcen der Organisation, auf denen die besonderen Stärken und Schwächen im Wettbewerb beruhen. Untersucht werden unmittelbar produktive sowie indirekt unterstützende Funktionsbereiche, wie die Finanzierung, das Personalwesen oder die Informationsverarbeitung. Die Analyse richtet sich vor allem auf relative Stärken und Schwächen im Vergleich zu den wichtigsten Wettbewerbern. Beispielsweise könnten das eigene Vorgehen beim Fundraising, die Gewinnung ehrenamtlicher Mitglieder oder die Sammlung und Speicherung wichtiger Informationen untersucht werden. Neben den Funktionsbereichen sollte sich die Analyse aber auch auf Querschnittsfunktionen wie die Effizienz, das Qualitätsniveau oder die Innovationskraft erstrecken. Wie in der Situationsanalyse sind Breite und Tiefe der Analyse den spezifischen Anforderungen in der jeweiligen Organisation anzupassen.

Der Ressourcenbegriff ist sehr weit gefasst. Er bezieht sich nicht nur auf das Finanzkapital, sondern auf alle materiellen und immateriellen Mittel, welche in die Leistungen der Organisation eingehen. Die folgende Abbildung zeigt einige wesentliche Ressourcengruppen (Osterloh/Frost 1996). 
Ressourcen

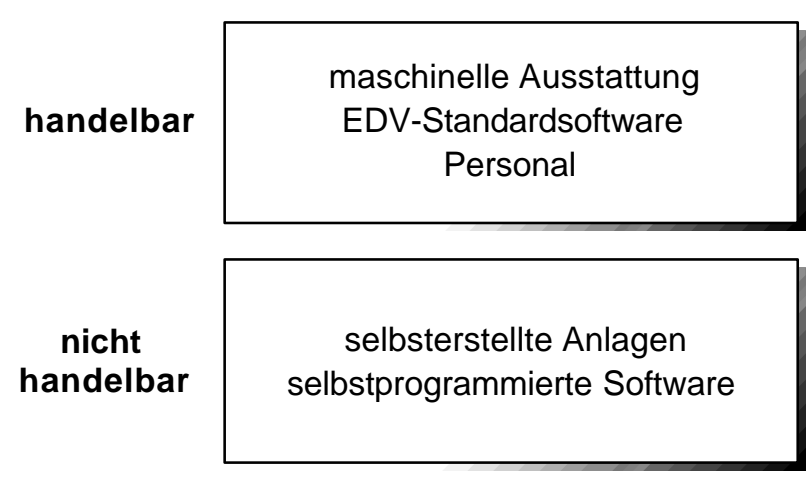

physisch nicht greifbar

\section{Lizenzen \\ Expertenwissen}

Organisationskultur

einzigartige Beziehungen

organisationsspezif. Qualifikationen

implizites Wissen

Situations- und Organisationsanalyse gehören eng zusammen und ergänzen sich gegenseitig. In den 70er und 80er Jahren hat die Strategielehre besonderes Gewicht auf die Situationsanalyse gelegt. Organisationsstrategien sollten vor allem positionsgerecht gestaltet sein. In jüngster Zeit ist aber eine zunehmende Verlagerung hin $\mathrm{zu}$ einer stärkeren Betonung der Organisationsanalyse erkennbar. Strategien sollen in erster Linie ressourcengerecht angelegt sein.

Ein Ergebnis dieser Verlagerung ist das Konzept der Strategischen Kernkompetenzen, das sich vor allem mit der Publikation von Hamel und Prahalad (1995) etabliert hat. Kernkompetenzen sind Fähigkeiten in einer Organisation, die es ihr ermöglichen, wesentliche Teile ihres Leistungsprogramms besser oder effizienter als die Wettbewerber zu erzeugen. Sie können sich auf Produktmerkmale (wie z.B. seine Qualität), aber auch den gesamten Ressourcenbereich erstrecken. Ziel des Strategischen Management ist es demnach, die Kernkompetenzen zu ermitteln und das Leistungsprogramm optimal darauf abzustimmen.

\subsection{Strategieentwicklung}

Je nachdem, welches Gewicht Situations- und Organisationsanalyse haben, ergeben sich unterschiedliche Ansätze für die Strategieentwicklung, wie die folgende Abbildung zeigt.

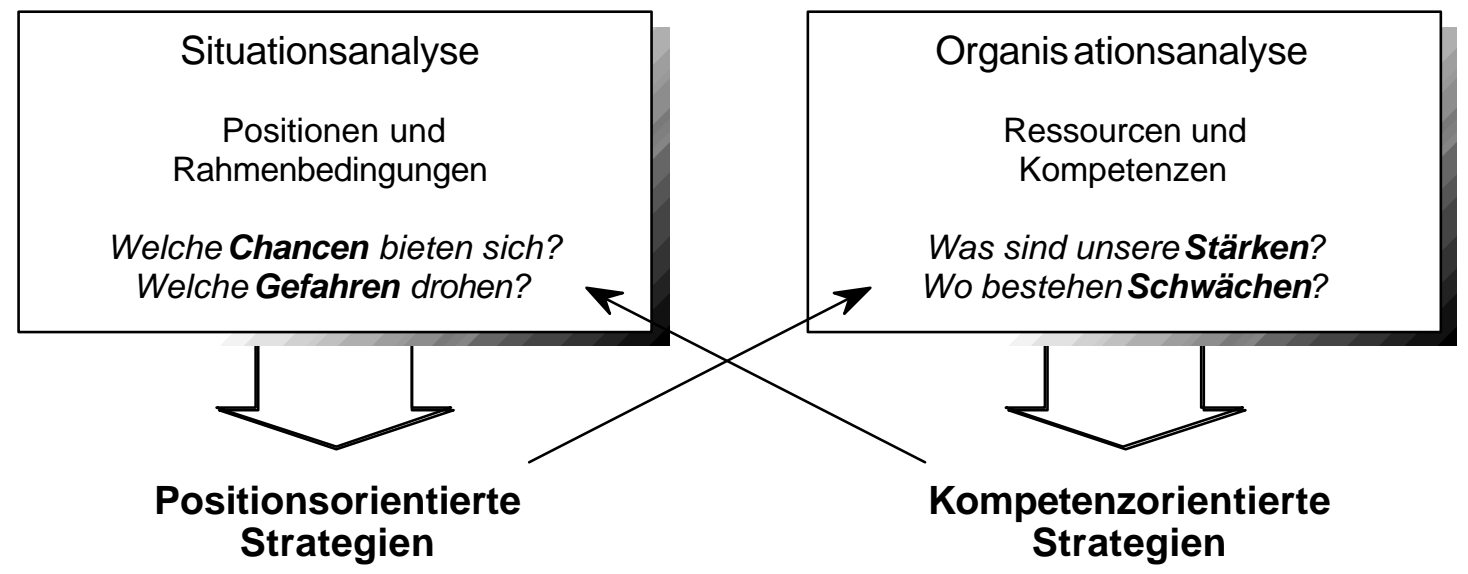

Positionsorientierte Strategien zielen darauf ab, sich unter gegebenen äußeren Bedingungen möglichst erfolgreich zu behaupten, attraktive Chancen zu nutzen und sich frühzeitig gegen Risiken zu wappnen. Ihr Ausgangspunkt ist die Situationsanalyse. Die Organisationsanalyse dient dazu, bestehende Defizite 
bezüglich der Ressourcen zu erkennen. Ihre Beseitigung ist ein wesentlicher Gegenstand des Maßnahmenplans zur Strategierealisierung. Setzt sich die Deutsche Bundesbahn beispielsweise zum Ziel, den Marktanteil im Fernverkehr gegenüber den Fluglinien auszuweiten, wird die Organisationsanalyse sich mit Defiziten im Schienennetz und dem Service auseinandersetzen müssen. Ergebnis ist die Einführung des ICE und in seiner Folge anderer Hochgeschwindigkeitszüge mit erweitertem Leistungsangebot.

Kompetenzorientierte Strategien sind darauf gerichtet, die vorhandenen Ressourcen optimal einzusetzen und dabei insbesondere die Kernkompetenzen möglichst vielfältig zu nutzen. Ausgangspunkt ist hier die Organisationsanalyse. Zu einer intensiveren Nutzung der eigenen Kernkompetenzen wird das bestehende Leistungsangebot verändert, variiert oder ausgeweitet. Gegebenenfalls werden auch Teilbereiche abgebaut, weil sie ohne Bezug zu den Kernkompetenzen sind. Die Ergebnisse der Situationsanalyse werden anschließend für eine Beurteilung der Wettbewerbschancen für das veränderte Leistungsangebot genutzt. Im Beispiel der Deutschen Bundesbahn könnten ressourcenorientierte Strategien dazu führen, das eigene Telekommunikationsnetz, das seit kurzem dem Telefondienstleister Arcor zur Verfügung steht, anderen Unternehmen für interne Kommunikationsnetze anzubieten. American Airlines verdient mit Flügen kaum mehr als mit Lizenzen für sein Buchungssystem, und der ehemals führende Reifenhersteller Dunlop produziert inzwischen alles mögliche aus Gummi - nur keine Reifen mehr. In all diesen Fällen stand die Frage nach einer verbesserten Ressourcennutzung vor der Marktanalyse.

Die beiden Strategietypen werden nur selten in der Reinform anzutreffen sein. In der Praxis sind Mischtypen wahrscheinlicher. Jede Form der Strategieentwicklung bewegt sich jedoch zwischen diesen beiden Extremen. Der Schwerpunkt verlagert sich im Wirtschaftssektor derzeit hin zu einer verstärkt kompetenzorientierten Herangehensweise, während der Nonprofit-Sektor die meist noch wenig entwickelte Situationsperspektive erschließen.

Ein traditionell weitverbreitetes Instrument zur Strategieentwicklung ist die Portfolio-Methode. Ursprünglich für die Analyse von Finanzrisiken entwickelt, wurde sie in den 60er und 70er Jahren von US-amerikanischen Consulting-Gesellschaften (BCG, McKinsey, Arthur D. Little) auf die Strategieberatung übertragen. Mit der Portfolio-Methode werden Strategische Empfehlungen aus zwei voneinander unabhängigen Faktoren abgeleitet:

- Chancen-Risiken-Faktor: Im Portfolio nach BCG ist es das Marktwachstum. Je höher das Wachstum ist, um so größer sind die sich bietenden Chancen, aber auch die Wahrscheinlichkeit zahlreicher Konkurrenten und damit verbunden der Aufwand, um die eigene Position halten zu können.

- Stärken-Schwächen-Faktor: Im Portfolio nach BCG ist es der relative Marktanteil, d.h. der Anteil im Vergleich zum größten Wettbewerber. Je größer dieser Wert ist, um so eher gelingt es einem Unternehmen, einen überdurchschnittlichen Ertrag zu erzielen.

Die Portfolio-Matrix lässt sich sowohl auf positionsorientierte als auch kompetenzorientierte Strategien von Nonprofit-Organisationen übertragen. Die folgende Abbildung zeigt dazu jeweils ein Beispiel. 


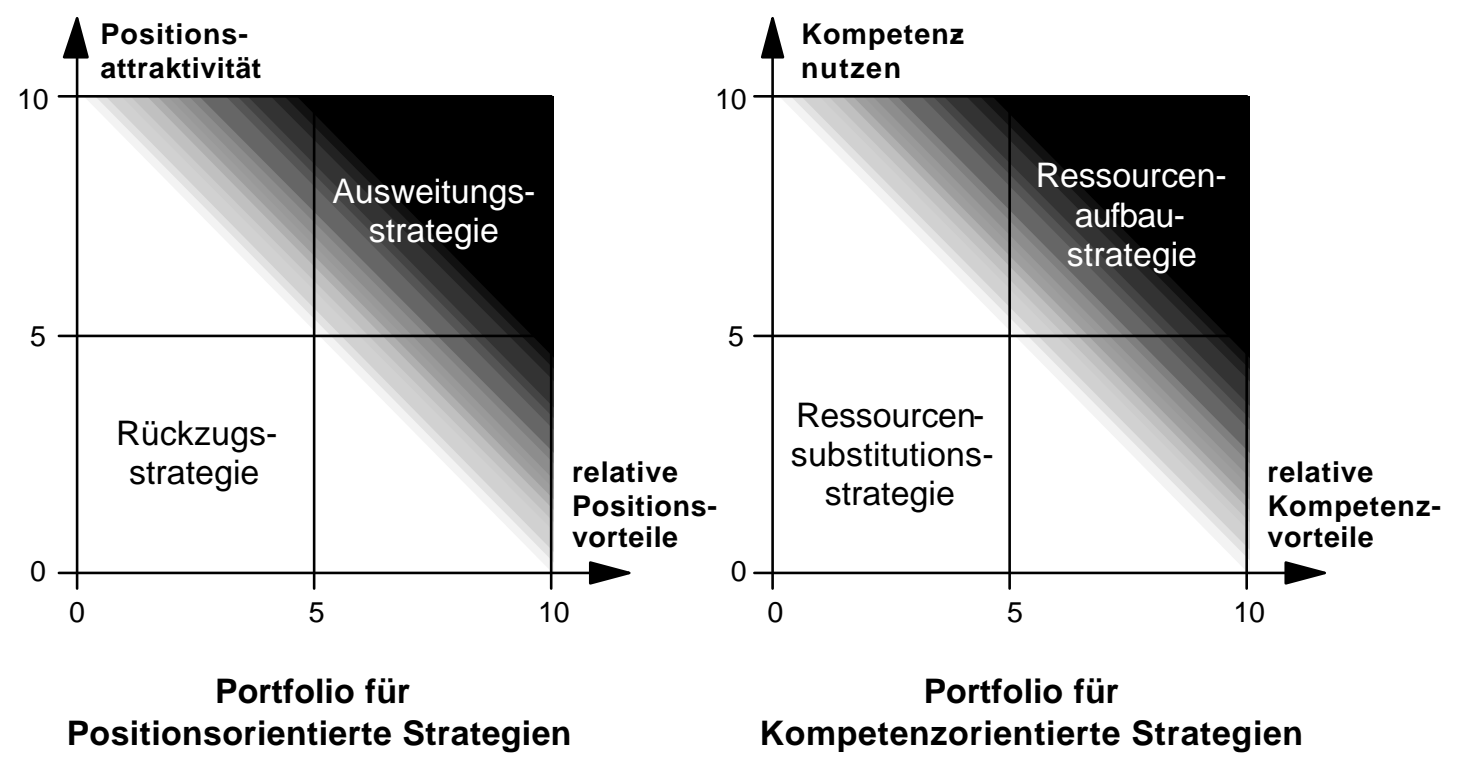

Zur Illustration gehen wir im folgenden Abschnitt von einer Hilfsorganisation aus, die auf private Spendengelder angewiesen ist und darum mit anderen ähnlichen Organisationen konkurriert.

Im Portfolio für Positionsstrategien werden die Elemente des Leistungsprogramms einer NonprofitOrganisation nach den Kriterien Positionsattraktivität und Positionsvorteile beurteilt und eingeordnet. In unserem Beispiel beziehen sich Positionen auf Gruppen von Spendern, von denen die Organisation bisher unterstiitzt wurde oder die sie noch auf sich aufmerksam machen könnte: z.B. politisch engagierte Männer und Frauen bis 30 Jahre, Personen über 65 Jahre, Katholiken, Familien usw.

- Positionsattraktivität: Die Attraktivität ergibt sich aus dem möglichen Spendenaufkommen der jeweiligen Gruppe. Relevante Kriterien sind beispielsweise Einkommens- und Vermögenslage, grundsätzliche Einstellungen oder die Erreichbarkeit über Medien. Die Attraktivität kann aber auch in Drittwirkungen bestehen, wenn beispielsweise bestimmte Gruppen auch eine höhere Neigung aufweisen, sich persönlich für eine Hilfsorganisation zu engagieren.

- Relative Positionsvorteile: Vorteile der eigenen Organisation können in bestehenden Bindungen zu den Gruppen, im Image der Organisation oder in einem engagierten Stamm von Fundraisern bestehen.

Je höher eine Position nach beiden Faktoren bewertet wird, um so eher empfiehlt sich nach der Portfolio-Methode eine verstärkte Bearbeitung der jeweiligen Gruppe (Ausweitungsstrategie), im anderen Extrem ergibt sich eine Empfehlung zum Rückzug, um die Ressourcen aus diesem Bereich erfolgreicher einsetzen zu können.

Im Portfolio für Kompetenzstrategien werden Ressourcen nach den Kriterien des Kompetenznutzens und der Kompetenzvorteile beurteilt. In unserem Beispiel könnten Maßnahmen zur Werbung von Spendern geprüft werden, unabhängig davon, ob sie von der Organisation aktuell eingesetzt, zukünftig geplant oder derzeit nur von konkurrierenden Organisationen in eigenen oder anderen Feldern (z.B. kommerzielle Produktwerbung) eingesetzt werden: z.B. Informationsstände in Fußgängerzonen, Plakate, Werbeanzeigen, Auftritte in politischen Diskussionen oder öffentliche 
Aktionen. Das Beispiel macht deutlich, dass eine Kompetenz, d.h. die Fähigkeit zur Durchführung solcher Maßnahmen, wiederum auf anderen Ressourcen beruht.

- Kompetenznutzen: Er ergibt sich aus seinem Einfluss auf das Spendenaufkommen. Relevante Kriterien sind beispielsweise die Reichweite, die Erinnerungsrate oder das Image der Maßnahme in der Öffentlichkeit. Darüber hinaus sind aber auch hier Drittwirkungen zu beachten, z.B. die Auswirkung auf die Bereitschaft öffentlicher Institutionen zur finanziellen Unterstützung der Organisation.

- Relative Kompetenzvorteile: Sie können beispielsweise in engen Kontakten der Organisation zu den Medien, einer hauseigenen Druckerei oder wiederum in engagierten Mitarbeitern begründet sein.

Je höher eine Kompetenz nach diesen beiden Faktoren bewertet wird, um so eher empfiehlt es sich nach der Portfolio-Methode, diese Kompetenz weiter auszubauen. Bei einer niedrigen Bewertung sollten die Möglichkeiten geprüft werden, sie gegebenenfalls durch andere Kompetenzen zu ersetzen Substitutionsstrategie).

Ziel der Portfolio-Analyse ist die Optimierung des eigenen Portfolio von Positionen bzw. Kompetenzen. Dazu muss der gesamte Aufwand für die Gewinnung von Positionen bzw. die Nutzung der Kompetenzen gegenüber dem Ertrag möglichst hoch sein. Das damit verbundene Risiko soll andererseits aber möglichst niedrig sein. In der Regel geht man von einem Spannungsfeld zwischen den beiden Teilzielen aus: Je größer der Nutzen ist, um so höher ist auch das Risiko in Wettbewerbssituationen. Risikoscheue Organisationen werden demnach eher weniger attraktive Positionen zu besetzen versuchen, während risikofreudige Organisationen nach möglichst attraktiven wenn auch gefährdeten Positionen streben werden. Entsprechendes gilt für den Aufbau von Kompetenzen.

Am Ende der Strategieentwicklung steht mit dem Strategischen Plan ein Katalog von Zielen, die innerhalb eines Zeitraums von zwei, drei oder fünf Jahren verfolgt werden sowie Maßnahmen, die dazu eingeleitet werden.

\section{$3 \quad$ Strategieverankerung}

Strategien sind Ideen von Zielen, die man erreichen will. $\mathrm{Zu}$ ihrer Verwirklichung müssen sie in der Organisation und ihren alltäglichen Routinen verankert werden. Die Strategieverankerung umfasst alle Maßnahmen, mit denen dazu beigetragen wird, dass die Mitglieder sowie Strukturen und Prozesse der Organisation zur Realisierung des Strategischen Plans beitragen. Sie können von der Umgestaltung des Leistungsprogramms oder der Schaffung neuer Aufgaben, über Informationsveranstaltungen und Schulungsmaßnahmen bis zur Entwicklung eines neuen Logo, mit dem die Organisation nach außen auftreten will, reichen. Große Bedeutung kommt den nachfolgend näher beleuchteten drei Aspekten zu: den Führungskräften in der Umsetzung von Strategien, der Organisationsstruktur sowie der Kultur, d.h. den handlungsleitenden Normen und Werten. 


\subsection{Die Rolle der Führungskräfte im Strategischen Management}

Im Strategischen Management ergeben sich für die Führungskräfte einer Organisation wichtige Vermittlungsaufgaben zwischen der Organisationsleitung und den dezentralen Bereichen. Sie sollen die strategischen Ziele weitergeben und sind für deren Realisierung im Tätigkeitsbereich jedes Organisationsmitglieds verantwortlich. Dabei lassen sich wiederum vier Phasen der Strategischen Führung mit spezifischen Führungsaufgaben unterscheiden.

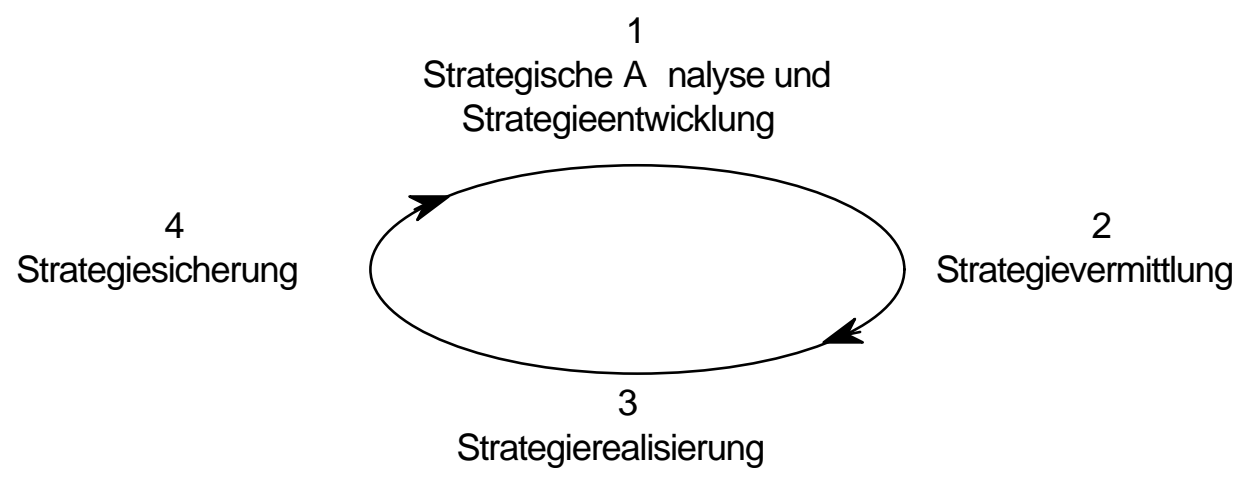

In allen Organisationen sind Strategische Analyse und Strategieentwicklung eine Kernaufgabe der Organisationsspitze. Unterschiede zeigen sich darin, welcher Personenkreis darüber hinaus in welcher Funktion und in welchem Umfang beteiligt wird: als Informationsquellen, als Berater oder als Mitentscheider. Die folgende Beschreibung der Führungsrollen geht von einem mittleren Partizipationsgrad aus.

- In der Phase der strategischen Analyse und Strategieentwicklung besteht die wesentliche Aufgabe der Führungskräfte darin, Informationen über die aktuelle Situation sowie die wettbewerbsrelevanten Stärken und Schwächen der Organisation zu sammeln und an die Spitze weiterzugeben. Die Führungskräfte nehmen die Rolle von Informationsknoten im Kommunikationsnetzwerk ein. Gegenüber ihren Mitarbeitern setzt diese Rolle nicht nur eine enge Beziehung voraus, sondern auch die Fähigkeit, sie dazu zu motivieren, wichtige Informationen und Erkenntnisse weiterzugeben.

- In der Phase der Strategievermittlung verändert sich die Führungsrolle grundlegend. Grundlage sind die strategischen Entscheidungen, welche zentral getroffen wurden und für alle Mitglieder von weitreichender Bedeutung sind, ohne dass sie daran aktiv beteiligt worden sind. Aufgabe der Führungskräfte ist es, ihre Mitarbeiter von den strategischen Entscheidungen zu überzeugen und ihnen deutlich zu machen, welche Rolle sie selbst in der Zielerreichung spielen. Dabei übernimmt die Führungskraft eine Vorbildfunktion, verkörpert in ihrem eigenen Verhalten die strategischen Ziele und konzentriert sich darauf, dass ihre Mitarbeiter sich ebenso mit ihren Teilzielen identifizieren. Dieser Führungsansatz versucht, mögliche Widerstände der Mitarbeiter gegenüber vorgegebenen Zielen durch die persönliche Überzeugungsfähigkeit der Führungskräfte zu überwinden.

- Ist ein grundsätzlicher Zielkonsens erreicht, rückt in der Phase der Strategierealisierung die Führungsaufgabe in den Vordergrund, in einem kooperativen Prozess mit den Mitarbeitern die notwendigen Veränderungen in ihrem Arbeitsbereich umzusetzen. Ein kooperativer und partizipativer Führungsstil unterstützt in dieser Phase die Identifikation der Mitarbeiter mit ihren Aufgaben am 
besten, da diese ihren persönlichen Beitrag möglichst eigenständig gestalten sollen. Trotzdem wird davon ausgegangen, dass die Führungskraft noch eine aktive Rolle einzunehmen hat.

- In der abschließenden Phase der Strategiesicherung, in der die notwendigen Veränderungen erreicht wurden, ist das wichtigste Ziel die Erreichung maximaler Effizienz in der Aufgabenausübung. Unter der Voraussetzung eines hohen individuellen Reifegrads der Mitarbeiter sind das Führungsprinzip der Delegation und ein Führungsstil, der Eingriffe auf Ausnahmesituationen beschränkt, als ideal anzusehen.

In vielen Nonprofit-Organisationen hat das basisdemokratische Prinzip ähnlich wie in selbstverwalteten Betrieben (Kück 1989) einen hohen Stellenwert. Dementsprechend hoch wird hier der Partizipationsgrad im Strategischen Management sein. Mit der Größe nimmt aber auch die Wahrscheinlichkeit zu, dass das Strategische Management zur Spezialaufgabe an der Organisationsspitze wird. Das erhöht die Bedeutung der nächsten Führungsebenen für die Umsetzung.

\subsection{Strategie und Organisationsstruktur}

Die Struktur vieler Nonprofit-Organisationen, wie sie im Organigramm dargestellt wird, spiegelt das Bemühen wieder, ihre Größe und innere Komplexität zu bewältigen. In einer strategischen Ausrichtung soll die Struktur jedoch vor allem dazu dienen, Wettbewerbsvorteile zu erzeugen und zu erhalten. Der Wirtschaftshistoriker Chandler hat bereits in den 60er Jahren in einer Untersuchung amerikanischer Konzerne festgestellt, dass mit zunehmender Komplexität der Unternehmensstrategien stets auch die Organisationsstruktur komplexer wurde. Daraus leitete er den Leitsatz "structure follows strategy" ab. Demnach ist die Organisationsstruktur immer wieder neu auf die aktuelle Strategie auszurichten.

Als Grundmodelle haben sich in der Organisationslehre die funktionale Einlinienorganisation, die divisionale Einlinienorganisation, die Matrixorganisation mit zwei überschneidenden Führungsebenen und das Stab-Linien-Modell neben einigen modernen Varianten (z.B. Netzwerkorganisation oder Prozessorganisation) und zahllosen Mischformen herausgebildet. Jedes Organisationsmodell beruht auf anderen Prinzipien der Arbeitsteilung und der Koordination. Daraus ergeben sich je nach Strategie andere Vorund Nachteile.

- Die funktionale Einlinienstruktur ist dadurch gekennzeichnet, dass jeder Bereich eine Fachaufgabe hat (z.B. Beschaffung, Auslieferung, Personalwesen, Informatik). In jedem Bereich ist Fachkompetenz gebündelt. Daraus ergibt sich als typische Stärke eine hohe Professionalität der einzelnen Funktionsbereiche. Sie bildet um so eher ein Strategisches Erfolgspotential, je homogener das Leistungsprogramm und je geringer die Veränderungsrate im Umfeld der Organisation sind. Die Spezialisierung schafft unter diesen Bedingungen auch günstige Voraussetzungen für eine starke Effizienzorientierung. Dieses Organisationsprinzip unterstützt hingegen spezifische Positionsstrategien für einzelne Tätigkeitsfelder der Organisation eher weniger gut.

- Die divisionale Einlinienstruktur beruht auf dem Prinzip, weitgehend autonome Organisationsbereiche zu schaffen, deren Aufgabe sich auf eine ganz bestimmte Gruppe von Adressaten, auf bestimmte Regionen oder ein bestimmtes Produkt beschränkt. Die meisten dazu notwendigen Funktionsbereiche sind dazu zusammengefasst und entsprechend dezentralisiert (z.B. 
Personal- oder Informatikbetreuer). Dieses Organisationsmodell bündelt leistungsprogrammbezogene Kompetenzen und unterstuitzt die Konzentration auf bestimmte Adressaten oder Regionen. Die funktionsspezifische Professionalisierung ist schwächer, die Fähigkeit, sich laufend den Veränderungen in spezifischen Positionen anzupassen, jedoch höher. Dieses Modell empfiehlt sich vor allem für größere Organisationen, in denen die einzelnen Bereich eigene und insgesamt sehr unterschiedliche Strategien verfolgen.

- In der Stab-Linien- und der Matrixstruktur wird auf unterschiedliche Weise versucht, die Stärken der beiden Einlinienstrukturen zu verbinden und die jeweiligen Schwächen abzubauen. Im StabLinien-Modell geschieht dies durch zentrale Stellen mit Koordinationsfunktion, in der Matrix durch eine zweite Führungsdimension. Jeder Mitarbeiter hat einen Fachvorgesetzten, ist gleichzeitig aber auch noch einer Führungskraft unterstellt, die alle Funktionen für ein bestimmte Produkt oder eine Region koordiniert. Erhofft werden dadurch Synergien. Es besteht jedoch die Gefahr, dass es vor allem zu Kompromisslösungen oder gar zu gegenseitigen Blockaden kommt. Die Konzentration der Kräfte und die Selektivität sind zentrale Grundlagen des Strategischen Management und geraten leicht in Konflikt zum Integrationsziel komplexer mehrdimensionaler Organisationsstrukturen. In der Regel erfordern diese Organisationsstrukturen einen höheren Aufwand zur Realisierung strategischen Erfolgs, bieten aber unter günstigen Bedingungen hohe Professionalität bei großer Flexibilität.

Es ist davon auszugehen, dass sich die meisten positionsorientierten Strategien besser mit einer divisionalen Struktur realisieren lassen. Kleinere autonome Einheiten sind anpassungsfähiger und können schneller auf Chancen und Gefahren reagieren. Eine kompetenzorientierte Strategie ist hingegen in aller Regel auf Expertise und Professionalität angewiesen, die sich in einer funktionalen Struktur leichter aufbauen lassen.

\subsection{Strategie und Organisationskultur}

Als Organisationskultur wird die Gesamtheit aller Weltbilder, Normen und Werte, Symbole und Gepflogenheiten bezeichnet, die für eine Organisation kennzeichnend sind und das Denken und Handeln der Mitglieder prägen. Dabei spielt es keine Rolle, ob die Kultur den Mitgliedern bewusst ist oder nicht. Die Kultur wird gelegentlich auch der "Stil des Hauses" genannt. Für einen Außenstehenden wird die Kultur dann erkennbar, wenn er als Kunde, neueingestellter Mitarbeiter oder Kooperationspartner in persönlichen Kontakt zur Organisation tritt. Sie kann sich ebenso in Umgangsformen und Redewendungen, Leitbildern und Überzeugungen aber auch Besonderheiten der Raumgestaltung oder Einrichtung äußern (Neuberger/Kompa 1987). Die Kultur hat eine wichtige stabilisierende und integrierende Funktion für eine Organisation, die sich in einer Veränderungsphase befindet. Sie bietet eine Grundlage für die Zusammenarbeit unterschiedlichster Personen und ist Teil ihrer gemeinsamen Identität.

Aus der Sicht des Strategischen Management kommt der Organisationskultur eine zweifache Bedeutung zu:

- Kultur ist eine Rahmenbedingung für die Strategieentwicklung und -umsetzung. Grundannahmen, Normen und Werte engen den Bereich denkbarer, erwünschter und für realisierbar gehaltener Strategien ein. Andererseits sind sie aber auch eine Quelle für strategische Ideen und für das Engage- 
ment unter den Organisationsmitgliedern, sie auch umzusetzen. Je besser die Strategie auf die Kultur abgestimmt ist, um so geringer sind die Reibungsverluste bei ihrer Realisierung.

- Kultur ist ein Gestaltungsfeld für die Strategieumsetzung. Wenn Strategien die bisherige Kultur in Frage stellen, müssen die dadurch erzeugten Konflikte aufgelöst werden. Strategien bleiben nie ohne Einfluss auf die Kultur, Aufgabe einer Kulturgestaltung ist es jedoch, den Prozess der StrategieKultur-Harmonisierung zu unterstützen.

Die Möglichkeiten und Grenzen der Kulturgestaltung und damit ihrer strategischen Ausrichtung hängen davon ab, wie das aktuelle Kulturprofil entstanden ist. Die Verankerung in einer über eine lange Zeit gewachsenen Weltanschauung, wie sie z.B. für Kirchen, Parteien oder Gewerkschaften kennzeichnend ist, beschränken die Möglichkeiten einer strategieorientierten Kulturgestaltung von vornherein. Innerhalb des verbleibenden Gestaltungsspielraums sind direkte und indirekte Maßnahmen zu unterscheiden:

- Direkte Maßnahmen zielen auf die sichtbare Oberfläche der Unternehmenskultur, d.h. auf Symbole. Sie reichen von der Veränderung von Strukturen und Ablaufprozessen über Leitbilder und Führungsgrundsätze bis zur Änderung von Hierarchie- und Funktionsbezeichnungen. Während damit gewissermaßen die äußere Hülle einer Organisation neu gestaltet wird, ist der daraus resultierende Einfluss auf die darunterliegenden handlungsleitenden Weltbilder, Normen und Werte ungewiss.

- Indirekte Maßnahmen zielen auf die Tiefenstrukturen der Organisationskultur. Mit Personalentwicklungsmaßnahmen oder Organisationsentwicklungsteams wird angestrebt, die meist unbewussten Normen und Werte offenzulegen und in Frage zu stellen, um damit einen partizipativen Prozess ihrer Weiterentwicklung in Gang zu setzen. Einige Organisationen haben versucht, durch eine neue Raumgestaltung die Kultur der Aufgabenerfüllung und Zusammenarbeit zu verändern. Schließlich spielen die Organisationsleitung und die mittleren Führungskräfte durch ihre Vorbildfunktion eine wichtige Rolle. Auch indirekte Maßnahmen sind nur einfache Impulse für den komplexen Prozess der Kulturveränderung, weshalb ihre Effekte immer ungewiss bleiben.

Da die Kultur einer Organisation von ihren Mitgliedern und insbesondere von den angestellten Führungskräften und Mitarbeitern getragen wird, kommt der Personalpolitik in der Kulturgestaltung eine wichtige Bedeutung zu (Klimecki/Gmür 1998). Das Qualifikationsprofil bestimmt nicht nur die Fähigkeiten und Fachkenntnisse, sondern es prägt Weltbilder und Werthaltungen. So rekrutieren z.B. Wohlfahrtsverbände zunehmend auch Betriebswirte für ihre Verwaltungstätigkeit, weil sie zunehmenden Finanzengpässen mit verstärktem Effizienzdenken begegnen wollen. Anreizsysteme umfassen alle materiellen wie nicht-materiellen Belohnungen, die eine Organisation vergibt. Sie prägen die Arbeitskultur, je nachdem, welche Kriterien beispielsweise für einen Aufstieg innerhalb der Organisation entscheidend sind: Zuverlässigkeit, Engagement, Leistung oder Loyalität?

Das Strategische Management bewegt sich in jeder Phase auf den Grundlagen von Führung, Struktur und Kultur. Sie bilden in der Organisation den Rahmen für die Analyse, Gestaltung und Umsetzung von Strategien. Als solcher sind sie ein beschränkender Faktor. Soll jedoch eine strategische Neuorientierung durchgesetzt werden, sind sie ein wichtiges Gestaltungsfeld. 


\section{$4 \quad$ Strategisches Controlling und Entwicklungsfähigkeit}

Controlling umfasst alle Funktionen zur laufenden Evaluation und Steuerung von Strategien in ihrer Umsetzung. Im Rahmen des Strategischen Management übernimmt es eine Korrekturfunktion, eine Antizipationsfunktion sowie eine Lernfunktion.

Die Korrekturfunktion besteht darin, Informationen über Abweichungen vom Strategischen Plan als Impuls für Korrekturhandlungen heranzuziehen. Dazu müssen im Vorfeld Toleranzgrenzen festgelegt werden, bei deren Überschreiten Korrekturmaßnahmen eingeleitet werden.

Die Antizipationsfunktion bezieht sich auf die Zukunftsorientierung der strategischen Kontrolle. Strategische Kontrollsysteme müssen nicht nur vergangenheitsorientiert sein, sondern auch antizipativ potentielle Planzielverfehlungen erkennen lassen. Da Plankontrollen nicht erst bei Vollendung des gesamten Plans durchgeführt werden, sondern bereits Teilrealisierungen überwacht werden, ist es besonders wichtig, zukünftige Anpassungserfordernisse zu identifizieren.

Möchte beispielsweise ein öffentliches Nahverkehrsunternehmen zur Erhöhung der Attraktivität ein Qualitätsimage aufbauen, könnte die Korrekturfunktion durch regelmäßige Befragungen zur Zufriedenheit der Nutzer realisiert werden. Damit würde die unmittelbare Wirkung von Änderungsmaßnahmen erfasst werden. Die Antizipationsfunktion könnte eine derartige Befragung ebenfalls ausfüllen, wenn aus der Entwicklung der Zufriedenheit auf einen Trend zu schließen wäre, auf den man sich in der Planung neuer Maßnahmen einstellen könnte.

Seit Anfang der 90er Jahre findet mit der Balanced Scorecard (Kaplan/Norton 1997) im Strategischen Management ein Instrument zunehmend Verbreitung, das sich insbesondere für ein Strategisches Controlling einsetzen lässt. Die Balanced Scorecard beruht auf der Annahme, dass Unternehmen zur Gewährleistung langfristiger Handlungsfähigkeit vier Managementbereiche optimieren müssen, die eng miteinander verzahnt sind. Für jeden der folgenden vier Bereiche sind klare Ziele und quantitative oder qualitative Kenngrößen zu bestimmen, um fortlaufend den Zielerreichungsgrad ermitteln zu können.

- Finanzen Hier geht es in erster Linie darum, den Erwartungen der Geldgeber gerecht zu werden. Die Rentabilitätsziele können durch Liquiditäts- oder Sicherheitsziele ergänzt werden.

- Kunden Dieses Managementfeld bezieht sich auf die kundenorientierte Gestaltung des Leistungsprogramms, um die finanziellen Ziele zu erreichen.

- Interne Prozesse: Für die angestrebten Kundenleistungen sind die internen Prozesse der Leistungserstellung entsprechend auszurichten.

- Lernen und Innovation: Schließlich soll die organisationale Lernfähigkeit gesteigert werden, damit die internen Prozesse reibungslos ablaufen.

Für die Anwendung auf das Strategische Management in Nonprofit-Organisationen ist der erste Bereich breiter zu fassen. An die Stelle der Ertragsmaximierung ist die allgemeine Erfüllung des wesentlichen Organisationszwecks zu setzen. In der folgenden Abbildung ist eine Balanced Scorecard mit den jeweiligen Grundfragen, Teilzielen und möglichen Kennzahlen abgebildet. Die Bestimmung der Teilziele und Kennzahlen richtet sich nach den Organisationsstrategien und den davon abgeleiteten operativer Handlungsprogrammen. Während die vier Managementfelder vorgegeben sind, müssen ihre jeweiligen Inhalte also erst noch strategieabhängig definiert werden. 
Die Balanced Scorecard ist mehrstufig: Sie wird in einem ersten Schritt für die Gesamtorganisation erstellt. Davon ausgehend werden für die einzelnen Teilbereiche der Organisation eigene Scorecards abgeleitet. Dieses Prinzip kann bis zur Ebene der einzelnen Teams weitergeführt werden. Der Nutzen dieses Instruments liegt in der einfachen Anordnung der vier zentralen Gestaltungsfelder, die sich beliebig ausdifferenzieren lassen - je nachdem, auf welche Aspekte in der jeweiligen Organisation besonderes Gewicht gelegt wird.

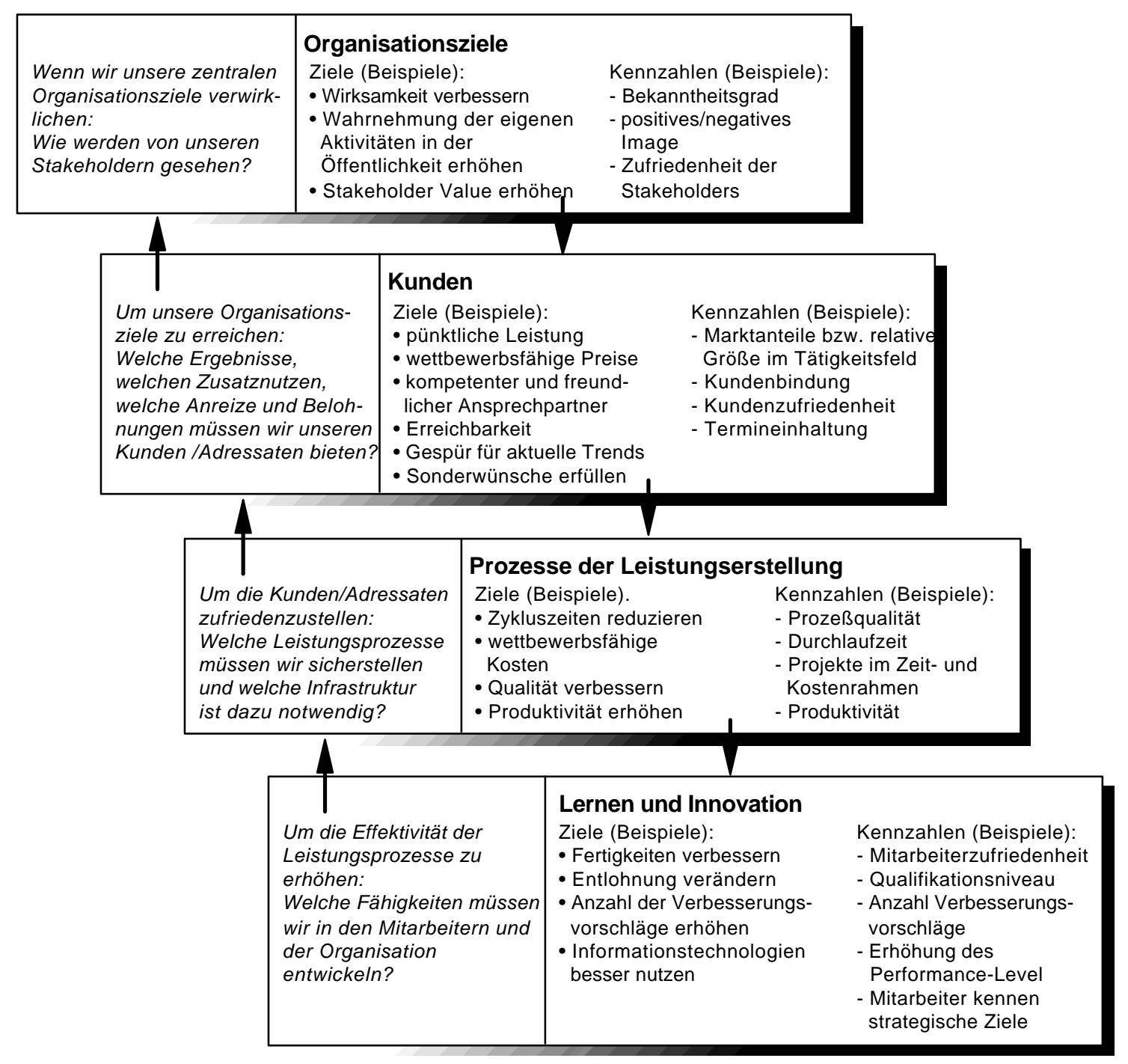

Die Lernfunktion des Controlling zielt auf die Erfahrungen von Planungs- oder Implementationsfehlern. So lässt sich aufgrund von Fehleranalysen für die Zukunft lernen. Der Lernprozess bezieht sich aber nicht nur auf eine verbesserte Planung und Implementation, sondern auch auf die Weiterentwicklung der strategischen Kontrollabläufe. Er kann sich auf drei unterschiedlichen Reflexionsniveaus vollziehen (Lombriser/Abplanalp 1997: 360). Dabei wird auf die Theorie organisationalen Lernens von Argyris und Schön (1978) zurückgegriffen, die drei Niveaus des kollektiven Lernens unterscheiden: 
- Als Anpassungslernen übernimmt das Controlling vor allem eine Korrekturfunktion. Aufgrund mangelhafter Ergebnisse werden die operativen Maßnahmen verändert. Lernen findet statt, indem diejenigen Maßnahmen fortgeführt werden, die sich für das gesetzte Ziel am besten eigenen, und diejenigen eingestellt oder ersetzt werden, die sich nicht bewähren. Beispiel: Eine Hochschule leitet immer wieder Marketingmaßnahmen mit dem Ziel ein, die Studienbewerberzahlen zu erhöhen.

- Als Veränderungslernen stellt das Controlling die strategischen Ziele und Grundsätze in Frage, auf deren Basis die operativen Maßnahmen eingeleitet wurden. Es findet statt, wenn ein Reflexionsprozess über die Ziele in Gang gesetzt wird, weil die eingeleiteten Maßnahmen immer wieder scheitern. Dazu könnte es in unserem Beispiel kommen, wenn die Bewerberzahlen trotz vielfältiger Aktivitäten zurückgehen und die Hochschule zur Erkenntnis gelangt, dass eine grundlegende Neugestaltung der Studienfächer viel größeren Einfluss auf das Wahlverhalten haben wird als einfache Werbemaßnahmen.

- Als Prozesslernen wird schließlich auch der Strategieentwicklungsprozess, aus dem Strategien und operative Maßnahmen abgeleitet wurden, hinterfragt. Nicht mehr die Inhalte von Zielen, Grundsätzen oder Maßnahmen werden in Frage gestellt sondern der Prozess ihrer Entstehung und Veränderung. Von Prozesslernen in unserem Beispiel könnte gesprochen werden, wenn die Hochschule ein Gremium einrichtet, das in regelmäßigen Abständen Untersuchungen über die aktuellen Ursachen des Wahlverhaltens von Schulabgängern durchführt, um so immer wieder neue Entscheidungsgrundlagen für die mittel- und langfristigen Ziele und Maßnahmen zu gewinnen.

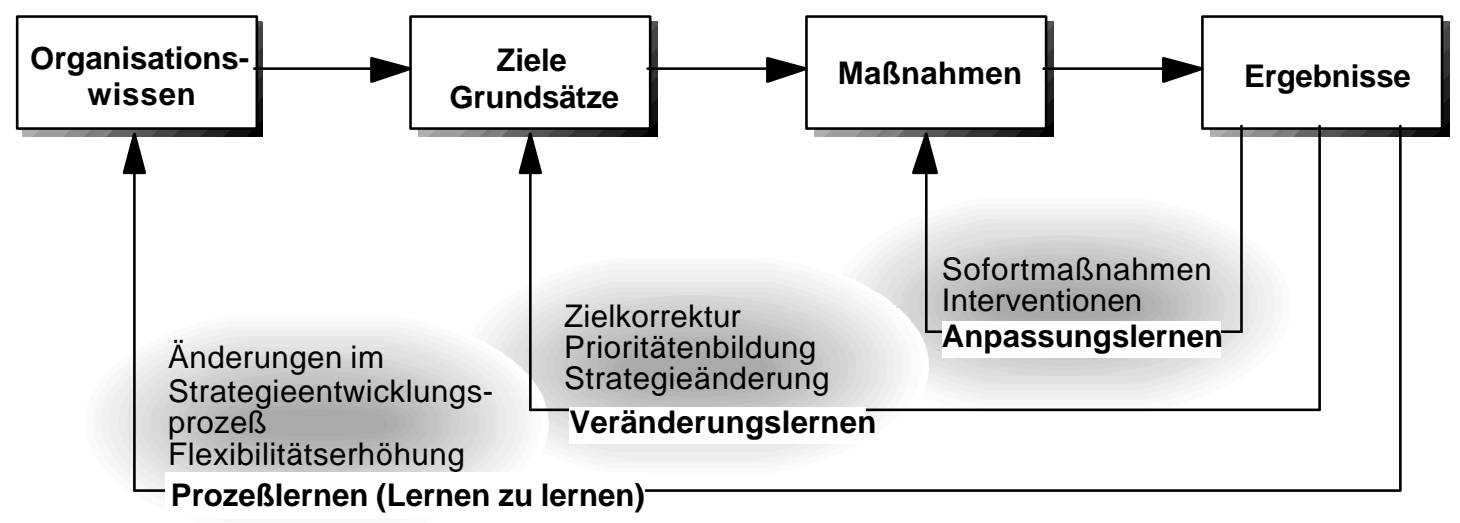

Insbesondere die Fähigkeit zum Prozesslernen ist eine wichtige Grundlage für die langfristige Entwicklungsfähigkeit einer Organisation. Diese erfordert ein Gleichgewicht zwischen Stabilisierung und Flexibilisierung unter der Voraussetzung komplexer und dynamischer Bedingungen. Strategisches Management setzt dabei den Rahmen und fortlaufende Impulse für die Veränderung von Organisationen (Gmür 1994). 


\section{$5 \quad$ Literatur}

Argyris, Chris/Schön, Donald (1978) Organizational Learning: A Theory of Action Perspective, Reading, Addison-Wesley.

Gmür, Markus (1994) Flexibilisierungsmanagement: Die Kunst der geschmeidigen Unternehmensführung, in: Socialmanagement 4 (4), S. 16 - 19.

Hamel, Gary/Prahalad, Coimbatore K. (1995) Wettlauf um die Zukunft, Wien, Überreuter.

Horak, Christian (1996) Stakeholder-Management für Nonprofit-Organisationen, in: Strunk, Andreas (Hrsg.) Dienstleistungscontrolling: Strategien zur Innovationssteuerung im Sozial- und Gesundheitssystem, Baden-Baden, Nomos, S. 87 - 103.

Kaplan, Robert S./Norton, David P. (1997) Balanced Scorecard: Strategien erfolgreich umsetzen, Stuttgart, Schaeffer-Poeschel

Klimecki, Rüdiger/Gmür, Markus (1998) Personalmanagement: Funktionen, Strategien, Entwicklungsperspektiven, Stuttgart, Lucius \& Lucius 1998.

Koteen, Jack (1997) Strategic Management in Public and Nonprofit Organizations, Westport, Praeger, 2. Auflage.

Kück, Marlies (1989) Betriebswirtschaft der Kooperative: Eine einzelwirtschaftliche Analyse kooperativer und selbstverwalteter Betriebe, Stuttgart, Poeschel.

Lombriser, Roman/Abplanalp, Peter A.(1997) Strategisches Management, Zürich, Versus.

Middleton Stone, Melissa/Crittenden, William (1993) A Guide to Journal Articles on Strategic Management in Nonprofit Organizations 1977 to 1992, in: Nonprofit Management and Leadership, 4 (2), S. 193 - 213.

Nutt, Paul C./Backoff, Robert W. (1992) Strategic Management of Public and Third Sector Management, San Francisco, Jossey-Bass.

Oster, Sharon M. (1995) Strategic Management for Nonprofit Organizations: Theory and Cases, New York/Oxford, Oxford University.

Osterloh, Margit/Frost, Jetta (1996) Prozeßmanagement als Kernkompetenz, Wiesbaden, Gabler.

Salipante, Paul F./Golden-Biddle, Karen (1995) Managing Traditionality and Strategic Change in Nonprofit Organizations, in: Nonprofit Management and Leadership, 6 (1), S. 3 - 20.

Thomas, James B./Clark, Shawn M./Gioia, Dennis A. (1993) Strategic Sensemaking and Organizational Performance: Linkages among Scanning, Interpretation, Action and Outcomes, in: Academy of Management Journal, 36, S. 239 - 270. 